\title{
STRATEGY OF SOLUTION FOR THE INVENTORY-ROUTING PROBLEM BASED ON SEPARABLE CROSS DECOMPOSITION
}

\author{
M. Elizondo-Cortés \& R. Aceves-García \\ Universidad Nacional Autónoma de México \\ Posgrado de Ingenieria, Circuito Exterior S/N, Ciudad Universitaria \\ Apdo. Post. 3000, Tel. 015556223282 ext. 128 Fax. 015556223282 ext. 107 \\ mareli@avantel.net
}

Received: January $28^{\text {th }}, 2003$. Accepted: May $17^{\text {th }}, 2005$

\section{ABSTRACT}

The Inventory-Routing Problem (IRP) involves a central warehouse, a fleet of trucks with finite capacity, a set of customers, and a known storage capacity. The objective is to determine when to serve each customer, as well as what route each truck should take, with the lowest expense. IRP is a NP-hard problem, this means that searching for solutions can take a very long time. A three-phase strategy is used to solve the problem. This strategy is constructed by answering the key questions: Which customers should be attended in a planned period? What volume of products should be delivered to each customer? And, which route should be followed by each truck? The second phase uses Cross Separable Decomposition to solve an Allocation Problem, in order to answer questions two and three, solving a location problem. The result is a very efficient ranking algorithm $\mathrm{O}\left(\mathrm{n}^{3}\right)$ for large cases of the IRP.

\section{RESUMEN}

El Problema de Inventario-Distribución (Inventory-Routing Problem, IRP), combina las actividades logísticas críticas de ruteo y manejo de inventarios. El objetivo es, al menor costo posible, atender las necesidades de un conjunto de clientes, utilizando una flotilla de vehículos que desde un almacén central, recorren rutas de distribución. El IRP es un problema NP-duro que en aplicaciones reales suele ser de gran tamaño. Para la solución de este problema se diseñó una estrategia conformada de tres fases, que responden a las preguntas características del IRP: 1. ¿A qué clientes atender en el horizonte de planeación? 2.¿Cuánto entregar a cada cliente? y 3. ¿Qué ruta debe seguir cada vehículo? La segunda fase, parte medular de la estrategia, utiliza la técnica de Descomposición Cruzada Separable para responder a las preguntas dos y tres, solucionando un problema de localización. El resultado es un algoritmo muy eficlente de orden $\mathbf{O}\left(\mathbf{n}^{3}\right)$ para instancias grandes del IRP.

KEYWORDS: Inventory, Routing, Cross Decomposition.

\section{INTRODUCTION}

Nowadays, many private and public organizations have realized that goods or services can be more valuable for a customer if there is good logistics management. Moreover, the availability of their product in the right place and at the right time, consistency in deliveries and ease in placing orders, are becoming importance aspects of the logistics services that form part of supply chains. Likewise, the important concept of customer value can be created through the avallability of the product in place and time by allocating orders, among other logistics services as part of the supply chain management. 
Vendor Managed Inventory (VMI) is an example of a combination of elements that can create a logistic value. Supplier-managed replenishment creates value for both the supplier and the customer. This is a win-win situation as the supplier saves on distribution costs and with the possibility of improving the coordination of deliveries, while customers do not have to use resources on inventory management. The transportation of the products and inventory management are the most expensive aspect of supply chains. Another reason for using supplier management replenishment is the rapid fall in the costs of the technology, making it possible to monitor customers' inventories offsite.

In spite of this, the reason VMI has not been more widely applied is attributed to the fact that it is extremely complicated to design a distribution strategy to minimize transport and inventory costs, while avoiding depleted inventory. This is called the Inventory Routing Problem (IRP). The first inventory-distribution model was developed by Federgruen and Zipkin [1] and is interpreted as an enriched vehicle routing problem (VRP), which includes inventory considerations. Numerous models and strategies have been proposed since then. The IRP is a "hard" problem, as many other logistic problems. These kinds of problems are called NP-hard so the optimal solution in a reasonable running time is almost impossible.

On the other hand, one of the major obstacles that prevents small and medium business from prospering in Mexico is the lack of awareness of and investment in logistics optimization projects. So it is necessary to look for alternative strategles in order to make these companies more competitive. This is why it is necessary to develop low-cost strategies of solution for logistics problems such as the IRP on a large scale.

In the next section, I make a quick review of the relevant literature about decomposition techniques; in Section 3 there is a detailed definition of the problem; in Section 4 the solution procedure is presented and this is tested in Section 5. Finally the conclusions are presented in Section 6.

\section{LITERATURE REVIEW}

The typical Inventory Routing Problem (IRP) Involves a central warehouse and a set of geographically-distributed customers. The model can be described as follows: Each customer possesses a known storage capacity (e.g. the tank size in the case of industrial gas).The central warehouse is responsible for maintaining a proper level of supply in the customers' depots and, as often as not, is at fault when there is a shortage. The distribution company makes all the decisions about delivery scheduled, on the basis of its estimates of the customers' demands and the known real-time levels of inventory, provided by electronic data interchange (EDI) systems.

A characteristic of the IRP is the simultaneous presence of two basic aspects: routing and inventory. The two aspects are combined in a dynamic environment, so that repeated decisions must be taken at different moments on the time horizon, and past decisions will influence future decisions. The decision-makers must decide:

when the deliveries should be made; i.e., when the vehicles should be loaded and when to visit the customers;

how much to load on each vehicle, in terms of the amount of each product under consideration, and how much of that load to distribute among the customers who require it;

- what route each vehicle should take to call on the customers.

Over time different methods have been developed to deal with these decisions, which are always present, though in distinct scenarios or formulations. Baita et al. [2] developed a classification of solution methods that groups these into two main types: 1 . Frequency domain methods, and 2 . Time domain methods. In the former the decision variables are the frequencies of replenishment or time between deliveries. In the latter, the delivery schedule is decided or, with discrete time models, the amounts and routes are decided, using fixed time intervals. 
There are three categories: Aggregation Methods, Fixed Policy Participation Methods and Methods for Discrete Frequency Models. In particular, the success of the aggregation method [3] is due to the fact that it produces analytical models that do not consume too many computer resources, although they do require skill for their construction and interpretation. The use of the aggregation method is more of an art than a completely formalized method, which can be a great advantage in specific cases. The Fixed Policy Participation Method only gives asymptotic optimality. In fact it can give bad or almost trivial solutions for small or medium-sized examples. Hall [4] showed that its main limitation is that the method cannot model coordinated deliveries, thus the results are only valid for independent deliveries, or can only barely be considered as upper bounds for the real costs. This limitation also occurs in discrete frequency models that, in this case, obtain extremely complex and unmanageable models.

\subsection{Time Domain Methods}

Contrary to frequency domain, this involves operations being programmed through a horizon that could be infinite. In principle, decisions are only taken once. Nevertheless the most interesting case is when, in a discrete time model, decisions are taken at the beginning of each time period (e.g. day or week), knowing the state of the system (inventory levels).

The time domain method is used in a number of papers by Dror and Ball [5], Dror and Lew [6], Dror and Trudeau [7], and Trudeau and Dror [8]. In each time interval only customers who have reached their inventory safety level are attended. Just one product should be delivered from one warehouse to several customers, whose demands, different In each time period, are deterministic in [5] and [6]; stochastic in [8]; stochastic or deterministic in [7]. More recently, Campbell et al. [9] demonstrates an application and a solution method, using a combination of the GRASP heuristic and integer programming, for a real application of industrial gas distribution for the company PRAXAIR. CousineauOulmet [10] applies the well-known metaheuristic Taboo Search to the IRP. However it was limited by the length of the planning period and, in fact, is considered to be in an initial stage of research.

\subsubsection{Decomposition Techniques}

As part of the time domain methods, the Decomposition Technique has given good results. This technique, which is used to solve large-scale problems as well as problems of linear programming with special structure, is characterized by a decomposition of the original system into subsystems, each one with a minor or separate sub-problem. The subproblems are considered manageable due to their size, but altogether they constitute a problem that exceeds the available computer capacity being immensely complex.

The principle of decomposition, including the primal and dual forms, is known as Benders Decomposition and Lagrangean Dual Decomposition (Dantzig-Wolfe), respectively. These techniques make it possible to take advantage of the special structure of the problem, by solving a sequence of simpler problems. Thus, the primal or dual substructure of the problem is explored. In fact, such strategies are considered dual for each other and in each one of them the cycling occurs between the master problem and the sub-problem. In particular, the decomposition techniques were proposed and used to solve the inventory routing problem (IRP), by authors such as Federgruen and Zipkin [1]; Chien, Balakrishnan and Wong [11] and Christiansen [12].

Van Roy [13] proposes a new decomposition method called Cross Decomposition for solving Mixed Integer Problem (MIP). This method is based on the relationship between Benders' and Dantzig-Wolfe's principles of decomposition. In fact, it is possible to establish that the Lagrangean dual sub-problem is a relaxed master problem in the Benders' decomposition and, at the same time, Benders' sub-problem can be considered a relaxed master problem for the dual decomposition. It is also a known fact that both methods are dual pairs, l.e., if Benders' algorithm is applied to a Linear Program (LP) problem, it coincides with the Dantzig-Wolfe decomposition algorithm applied to the dual of that same LP problem [14]. 
The basic fundamental idea of Cross Decomposition is to use both sub-problems in a single solution procedure, exploiting the fact that in some cases, both sub-problems are simple to solve. Van Roy [13], applied Cross Decomposition to a set of standard MIP test problems and obtained a $20 \%$ average reduction in solution time combined with the best aldorithms known at the time.

\section{DEFINITION OF THE INVENTORY ROUTING PROBLEM (IRP)}

The definition of the problem was as follows: A single product (boxes, containers) is distributed from a warehouse to several customers in different geographic locations. The geographic position of the warehouse and the customers is known, as well as the distances between the warehouse and the customers and the distances between all the customers. Cost parameters (storage and organization costs) are used to characterize the customers. It is assumed that every customer's demand for the product is known and constant in time, in fact, the real time needs are known, which allows for a push-system strategy, based on the precise and timely knowledge of all the inventories. The deliveries begin at the start of every one-day period, using vehicles characterized by a given capacity, fixed costs and variable costs in regards to distance covered. There is no limitation in the central depot's supplying capacity. A mixed fleet of vehicles is avallable to take care of the customers in the time period. There are limitations in the capacity of the vehicles and their size is a decision variable that will be involved in the optimization process. Time is the decision domain for the problem, as it defines fixed time periods of one day. Moreover it is assumed that the customer's daily demand does not exceed their storade capacity.

The alm is to meet the customers' demand at the lowest cost, comprising inventory-related costs, including storage costs, together with fixed supply costs; and distribution costs, including fixed costs plus the costs involved in the distanced covered by the vehicles used. So, the problem is to find the supply and distribution policies, defined on the basis of the answers to the four following questions that must be answered at the start of each day:

Which customers must be supplied that day?

2. What vehicle will be used to supply each customer?

3. What volume of the product is to be delivered to the customers when visited?

4 What deliverv route will each vehicle take?

\section{PROPOSED STRATEGY}

The inventory routing problem (IRP) is hard-NP for a context of NP-completeness, as the vehicle-routing component of the problem, which now, moreover, includes inventory restrictions, is NP-hard and generalizes the Traveling Salesman Problem (TSP) [10]. In fact, all the non-trivial vehicle-routing problems are NP-hard [15], so it is unlikely that an algorithm of polynomial time can be developed to its optimal solution. So, a three-phase strategy, which responds to the four questions, was designed for the solution of the treated IRP and is as follows:

Phase I answers the question: 1. Which customers must be supplied that day?, by applying an Optional Replenishment System (ORS), that forces the review of the inventory level at a fixed time frequency and orders restocking if the level has fallen below a certain amount. Thus, this is basically a fixed time period model. A demand level for that day $D$ is forecast from historical data about daily demand. As supplying stock involves time and economic resources, a minimum order size $Q$ can be established. A good option for calculating $Q$ is to use the Economic Order Quantity (EOQ) equation. Therefore, when the product is checked, the inventory position $I$, is subtracted from the required level of restocking $D$ and the result is called $q$. Formally establishing 


$$
q=D-I
$$

where

$D$ : level of forecast demand
$I$ : current inventory level
$a$ : shortfall for achieving the maximum level of inventory
$Q:$ amount of the acceptable minimum order

and the rule is that only if $I \leq Q, q$ is ordered; if $q=0$ the customer is not served. Using an ORS for each customer, depending on their specific circumstances, the supplier decides if they should be supplied on that day or wait for the next review. Supply, the subgroup of customers who will be visited that day can be obtained. The information required during this phase consists in the specific $D, I, q$ and $Q$ for each customer depending on their demand, capacity, inventory levels, restocking costs, shortfall and storage. Once the question is answered, you go on to the next phase.

Phase II responds to the questions: 2 . What vehicle will be used to supply each customer? and 3 . What volume of the product is to be delivered to the customers when visited? These questions are answered by applying a decomposition technique called Separable Cross Decomposition [16], to a Facility Location (FL) problem that, by analogy, assumes that the services are vehicles that will deliver to the customers. The use of Separable Cross Decomposition contains a result that is fundamental to the study of the structure of the problem, by taking advantage of the research started by van Roy [13], solving a FL problem that uses the binary variables to allocate vehicles to customers.

It is therefore the problem of Allocation of Vehicles and Distribution (AVD) with the following formula: where

$m \quad$ number of available vehicles

$$
\text { number of customers }
$$

$d_{j} \quad$ customer demand $j$

$f_{i} \quad$ fixed cost for the route $i$

$a_{i} \quad$ : vehicle capacity $i$

$c_{i i} \quad$ cost of distribution to customer $j$ vehicle $i$, the cost is determined as a function of distance

$x_{i i} \quad$ fraction of the customer's total demand that has been supplied $j$ using vehicle $i$

$y_{i}=1$ if vehicle $i$ is used, 0 if not

The restriction (3.1) ensures that the demand is totally supplied, (3.2) establishes distribution only with active vehicles, (3.3) considers the use of enough vehicles to attend to the demand and (3.4) avoids exceeding the capacity of the vehicle.

There are some difficulties in optimizing these types of problem, because of their size and combinatorial structure. In fact, logistic-type problems such as the IRP and AVD are usually very big considering their large number of variables and restrictions. The AVD in itself is very complicated, since the single basic decision to use or not to use a vehicle gives problems a complex combinatorial structure. When it is a question of problems that associate that combinatorial 
structure with a great size, the complications increase, which is why mathematical programming relies on key techniques for this type of challenge such as bounding techniques, duality theory and decomposition techniques [16].

This problem, in particular, has two types of inherent deçsions: choosing the vehicles to be used and the best way to supply the customers. This makes decomposition techniques an attractive option for dealing with it, thus when the discrete decision of choosing the vehicle has been done, the ongoing distribution problem is, in general, easier to solve. Even if it is not possible to take advantage of this characteristic in the design of solution algorithms, decomposition can still be a very attractlve option, if the Allocation of Vehicles and Distribution (AVD) problems were not complicated by the discrete decision of choosing services and were formulated as a linear programming problem (relaxing the integrality restrictions for the problem's variables), it would still be very big and difficult to solve. Fortunately this problem has a special structure that can be exploited by decomposition techniques [16].

As a result of Van Roy's research [17] and the structure of the AVD, we can confirm that when the cross decomposition strategy is applied to this problem, a consequence solution is produced for two sub-problems, incorporating a ping-pong process between them, reducing the number of master problems to be solved. Three facts are fundamental for the Cross Decomposition algorithm:

the relationship between the primal (Benders) and the dual (through Lagrangean relaxation) decomposition;

2. the sub-problems $\left(S P x_{2}\right)$ and $\left(S D u_{2}\right)$ can be considered master problems for each other;

3. considerations under which $(P)$ can be solved by only iterating between both sub-problems.

The research developed by Aceves [16], incorporates into this cross process the strategy of Lagrangean Separable Relaxation, a special Lagrangean Relaxation case that is very advantageous because, with this scheme, none of the original restrictions disappear and it is not necessary to choose between the quality of the bound obtained and the degree of difficulty of the problem that remains. When incorporated, It establishes that it is not necessary to use the master problem in the solution, i.e., $(P)$ can be solved just by iterating between sub-problems, completely avolding the master problems. This procedure is called Separable Cross Decomposition and is the procedure applled to the Allocation of Vehicles and Distribution (AVD) problem in this phase.

Two sub-problems are obtained when Separable Cross Decomposition is applied to the AVD problem. The application of Benders to the primal gives us:

Minimize $\sum_{i=1}^{m} \sum_{i=1}^{n} c_{i j} x_{i j}+\sum_{i=1}^{m} f_{i} y_{i}$

subject to

$$
\begin{array}{cc}
\sum_{i=1}^{m} x_{i j}=1, & \forall j \\
\sum_{j=1}^{n} d_{j} x_{i j} \leq a_{i} y_{i}, & \forall i \\
0 \leq x_{i j} \leq y_{i}, & \forall i . i
\end{array}
$$

that is a transport problem; and applying the Lagrangean Separable Relaxation to the dual problem gets the subproblem 


\section{$\underset{x}{\operatorname{Minimize}} \sum_{i} \sum_{j}\left(C_{i j}-\lambda_{i}\right) x_{i j}$}

subject to

$$
\begin{aligned}
& \sum_{i} x_{i j} \geq d_{j}, \quad \forall j \\
& \sum_{j} x_{i j} \leq a_{i}, \quad \forall i \\
& x_{i j} \geq 0, \lambda_{i}<0, \quad \forall i, j
\end{aligned}
$$

with values of $\lambda_{i}$, for $i=1, \ldots, m$ with

$\lambda_{i}^{\circ}=-\frac{f_{i}}{a_{i}}, \forall i$ as initial value, and

$\lambda_{i}^{k}=\frac{f_{i}}{\sum_{j} x_{i j}}, \forall i$ for the subsequent values.

This result has made it possible to develop an algorithm that is much simpler that those previously obtained [16].

\subsection{Separable Cross Decomposition Algorithm}

Using the primal $S P_{y}$ and dual $S D_{\lambda}$ sub-problems, iterating between their solutions by fixing the values of the primal $y_{i}$ or dual $\lambda_{i}$ varlables and starting from an assumption for such variables, it is possible to go from one to another of the following stages:

1) fix $y_{i}$ at its current value and solve the Benders sub-problem $S P_{y}$, that is a transport problem, in order to generate a new value for the upper bound of $(P)$ and

2) fix $\lambda_{i}$ at its current value and solve the Lagrangean sub-problem $S D_{\lambda}$, to generate a new value for the lower bound of $(P)$. The following are stages of the algorithm:

$$
\begin{aligned}
& v_{D}(-\infty), v_{P}(+\infty) ; Y_{i}^{0}=1, \text { for } i=1, \ldots, m ; \\
& \lambda_{i}^{0}=-\frac{f_{i}}{a}, \text { for } i=1, \ldots, m .
\end{aligned}
$$

2.- Solve. Dual sub-problem $S D_{\lambda^{k}}$ (transport problem) to obtain $y_{i}^{1}=1, \sum_{j} x_{i j}$ y $v\left(S D_{\lambda^{k}}\right)$

2.1.-Calculate. $\lambda_{i}^{k}=-\frac{f_{i}}{\leftarrow}$, for $i=1, \ldots, m$.

3.- Test. If $\lambda_{i}^{k-1}=\lambda_{i}^{k}$ for $y_{i}^{k}=$, then end. Else, Identify which $y_{i}^{k}=$ for $i=1, \ldots, m$ 
Solve. Sub-problem primal $S P_{y_{i}^{k}}$ (transport problem), to obtain $v\left(S P_{y^{k}}\right)$

5.- Test. If $v\left(S P_{v^{k}}\right) \quad v\left(S D_{\lambda^{k}}\right)$, then end. Else, return stage 2 but now with $\lambda_{i}^{k}$

An analysis of the convergence of the Cross Decomposition algorithm, developed by Holmberg [18], Indicates that the cross decomposition algorithm has finite convergence for problems where the Dantzig-Wolfe decomposition algorithm or the Benders decomposition algorithm have finite convergence. This result is very advantageous as the reason for using cross decomposition is its practical efficiency, l.e., it is developed to have a quick convergence in practice, by replacing the master problems with easier sub-problems, as much as possible. Thus it is necessary to see if the algorithm used to solve the transport problems has finite convergence.

In subsequent iterations of the Separable Cross Decomposition, the most economic routes gradually get cheaper and, in consequence, they are allocated greater flow until they are saturated, elther by using up the capacity of the vehicle or meeting the demand, i.e.:

$$
\sum_{i} x_{t j}^{k-1} \leq \sum_{i} x_{t j}^{k} \leq \sum_{i} x_{t j}^{k+1} \leq \ldots \text { with } \lambda_{t}^{k-1} \leq \lambda_{t}^{k} \leq \lambda_{t}^{k+1} \leq \leq-\frac{f_{t}}{a_{t}}
$$

Thus, the vehicles that satisfy the demand obtained from the solution of the Lagrangean dual sub-problem $S D_{\lambda}$ can be used as active vehicles; i.e. $y_{i}=1$, with $i=1, \ldots, m$, that were fixed in the primal sub-problem $S P_{y}$.

So the separable cross decomposition algorithm ends in an finite number of iterations and obtains the solution to the Facility Location (FL) problem of Phase II. Thus it is known: which vehicle looks after each customers and what amount of product should be delivered to them. From this result you go on to the next phase.

Phase III responds to the question: 4. What delivery route will each vehicle take?, which is answered by solving a Traveling Salesman Problem (TSP), for each of the vehicles used. The proposed algorithm is the Adaptation-Prim-2OptHybrid heurlstic method, whose components are the 2Opt Method and an Adaptation of Prim's Algorithm Method. In order to minimize the total amount of distance, the 2Opt Method improve successively an initial route. This is done by eliminating 2 edges in each iteration and reconnecting the route with new edges. The Adaptation of Prim's Algorithm Method obtains an initial route with a minimum spanning tree using a greedy algorlthm. The use of Adaptation-Prim$2 \mathrm{Opt}$-Hybrid heuristic method obeys the initial supposition of a great number of customers for each vehicle to visit. Castañeda [19] has an interesting study where she compares heuristic and exact methods for the TSP and proposes the aforementioned hybrid as highly advantageous in respect to other heuristics, for very large TSP cases. According to Castañeda [19], the idea of hybridization arose as a result of the 2Opt theory mentioning that this method is better when commenced with a good route. So the Adaptation of Prim's Algorithm was decided on to find this initial route that would be used by the 2-Opt method, thus obtaining the Adaptation-Prim-2Opt-Hybrid method.

\section{EFFICIENCY OF THE STRATEGY}

In the idealization of the strategy, the need to define algorithms, that would solve more manageable problems in an easy and feasible way, was ever present. As it has already been mentioned, the essential part of the strategy is the application of Separable Cross Decomposition in Phase II. Thus an algorithm is obtained that produces a running solution for two transport sub-problems. It is interesting to note the enormous advantage of having this type of problem, as their solution algorithms have a low. order of complexity, $\left.\mathbf{O}^{\mathbf{n}^{3}}\right)$ [20] and moreover, any type of commerclal software can be used to solve them, even general purpose language programs. In such a way that the order of the proposed strategy is $\left.\mathrm{O} \mathrm{n}^{3}\right)$, which makes it extremely efficient. 
In order to assess the strategy's efficiency, 9 problems were constructed: 4 small problems with less than 10 customers, 2 medium-sized ones with 30 and 35 customers respectively and tree large problems with 150, 200 and 250 customers. To the proposed strategy, a direct computational comparison of the effectiveness of the results presented in this paper is not possible. In fact, each author either considered a slightly different problem or solved a completely different set of instances. However, we show the characteristics of the test instances and included in table I are the values computed respect to the optimal, whenever it was possible.

The fixed costs, capacitles and demands were taken from the examples given in Aceves [16]. The data generated as random variables were the customers' demands and the matrices for distance (dense matrices) between customers and between customers and the central warehouse. Said variables were obtained by means of the statistical package STATGRAPHICS. The capacities of the vehicles $\left(\mathrm{m}^{3}\right)$ took the values $24,16,8$ and 4 . The fixed costs varled between 50 , 40, 35, 30, 25 and 20. Every problem was solved by obtaining the optimum using the Branch and Bound (B-B) algorithm, by means of the LINGO package. Afterwards each problem was solved with the proposed algorithm, by means of a computer program developed in Pascal. The computer system used was a Pentium 4, at 2.00 Ghz with 256 MB RAM.

Table 1. Computational results

\begin{tabular}{|c|c|c|c|}
\hline Problem & Number of customer-vehicles & Proposed strategy & Optimal solution \\
\hline$E 005-03$ & $4-3$ & 9819 & 9819 \\
\hline$E 006-04$ & $5-4$ & 16724 & 16724 \\
\hline$E 008-02$ & $7-2$ & 21256 & 21256 \\
\hline$E 009-04$ & $8-4$ & 13114 & 13114 \\
\hline$E 031-09$ & $30-9$ & 4332 & 4332 \\
\hline$E 036-11$ & $35-11$ & 3120 & 3120 \\
\hline$E 151-08$ & $150-8$ & 14770 & Unknown \\
\hline$E 201-16$ & $200-16$ & 44586 & Unknown \\
\hline$E 251-20$ & $250-20$ & 72123 & Unknown \\
\hline
\end{tabular}

The results obtalned with the proposed strategy were: 1 . an extremely short solution time, as three iterations at most were used to solve the problems and, in every case, the results were obtained in less than 3 seconds, however; the optimal values obtained of E031-09 and E036-11 were 28 and 35 days respectively; 2 . the quality of the solutions was excellent in small and medium size problems, in fact, the optimum was obtained.

\section{CONCIIISIONS}

Inventory Routing Problem (IRP) modeling is relatively new and it is not yet possible to say that there is a standard general formulation. A ranking strategy $\mathbf{O}\left(\mathrm{n}^{3}\right)$ was obtained that is easy to implement and apply. It gives good quality results and is efficient in computational effort even for large problems, for obtaining the solution to an essentially NPnard problem, the general goal of this research paper. This strategy's low order of complexity makes it much more efficlent than the methods used to solve the IRP, which were reviewed in the state of the art and solved cases, that coincided with the one dealt with in this study. Thus, this comparison demonstrated the superiority of the proposed strategy as it can handle bigger problems with less computational effort. It is necessary to promote and continue esearch into the methodology for an optimal solution to different instances of the problem and reduce their computational complexity.

yystems optimization is to be recommended as an approach that can -considering the economic, social, political and echnological challenges that decision-makers are faced with- make businesses more competitive. Competitiveness is 
important for every type of organization and enterprise that wants to be up-to-date and successful in their sphere of action and contributes not only to their own economic development but also to that of their surroundings.

\section{REFERENCES}

Federgruen, A. and Zipkin. P. A comprised vehicle routing and inventory allocation problem. Operations Research; 32, pp.1019-1037, 1984

Baita, F., Ukovich, W., Pesenti, R. and Favaretto, D. Dynamic Routing-And-Inventory Problems: A Review. Transportation Research; 32: 8, pp.585-598, 1998

Daganzo, C. Logistics System Analysis. New York, Springer Verlag, pp. 10, 1996.

Hall, R. W. A note on bounds for direct shipping cost. Management Science; 38, pp. 1212, 1992.

Dror, $M$. and Ball, $M$. Inventory/routing: reduction from an annual to a short-period problem. Naval Research Logistics; 34, pp. 891-905, 1987.

Dror, $M$. and Lew, L. A vehicle routing improvement algorithm comparison of a greedy and a matching implementation for inventory routing. Computers and Operations Research; 13, pp. 33-45, 1986.

Dror, $M$. and Trudeau, P. Cash flow optimization in delivery scheduling. European Journal of Operational Research; 88, pp. 504-515, 1996

Trudeau, P. and Dror, M. Stochastic inventory routing: route design with stockouts and route failures. Transportation Science; 26, pp. 171-184, 1992.

Campbell, A., Clarke L., and Savelsbergh M. Inventory Routing in Practice. The Vehicle Routing Problem. Eds. P. Toth and D. Vigo. Philadelphia: Society for Industrial and Applied Mathematics, pp. 309-330, 2002.

Cousineau-Ouimet, K. A Tabu Search Heuristic for the Inventory Routing Problem. Artículo no edltado. Department of Quantitative Methods, École des Hautes Études Commerciales. Montreal, Canada 2002.

Chien, W., Balakrishnan, A. and Wong, R. An integrated Inventory allocation and vehicle routing problem. Transportation Science; 23, pp. 67, 1989

Christlansen, $M$. Decomposition of a Combined Inventory and Time Constrained Ship Routing Problem. Transportation Science; 33, pp. 3-16, 1999

Van Roy, T. J. Cross decomposition for mixed integer programming. Mathematical Programming;25, pp. 46-63, 1983.

Lasdon, L.S. Optimization Theory for Large Systems. USA: MCMillan Series in Operations Research, pp. 381 , 1970.

Bramel, J. and Simchil Levi, D. A location based heuristic for general routing problems. Operations Research; 43, pp. 649-660, 1995.

Aceves García, R. Un Algoritmo para Resolver el Problema de Localización de Servicios con Restricciones de Demanda y Adicionales. Tesis Doctoral (Investigación de Operaciones), México: DEP-FI, UNAM 1996.

Van Roy, T. J. A cross decomposition algorithm for capacitated facility location. Operation Research Society of America; 34, pp. 145-163, 1984.

Holmberg, K. On the Convergence of Cross Decomposition. Mathematical Programming; 47, pp. 269-296, 1990.

Castañeda Roldán, C. Estudio comparativo de diversos métodos de soluclón del problema del agente viajero (PAV). Tesis de Maestría en Ingeniería en Sistemas Computacionales, Puebla: Universidad de las Américas 2000.

Toth, P. and Vigo, D. The Vehicle Routing Problem. SIAM Monographs on DIscrete Mathematics and Applications. Philadelphia: Society for Industrial and Applied Mathematics, pp.134, 2002. 
Mayra Ellzondo Cortés. She obtained a Master in Sclences Degree in Operational Research at the National Autonomous University of Mexico (UNAM), and a PhD in Operational Research at the same university. Her research interest Include Optimization, Simulation and Logistic. She is a Teacher and Researcher in the Engineering Graduate School of the UNAM.



Ricardo Aceves Garcla. Dr. Aceves studied Chemical Engineering at the Autonomous University of Puebla, Mexico, and obtained a Master in Sciences and a PhD. in Operational Research at the Engineering Faculty of the National Autonomous University of Mexico (UNAM). He has been working in various projects in the field of Transportation and Operational Research, both public and private organizations. At present, he is a full time Teacher and Researcher at the Engineering Faculty and Engineering Graduated School of the UNAM, and he is a president of the Mexican Institute of Systems and Operational Research. 